$10-14 \%$ and 10 had $\geq 15 \%$. The median age at presentation was 5 days (2-12 days), median sodium level with $>15 \%$ weight loss babies was 152 (143-162).

Conclusions A significant number of neonatal re-admissions were due to abnormal weight loss of $\geq 10 \%$ and majority were due to failure to establish breastfeeding. An earlier audit in 2009 had identified need for more breast feeding support. Although systems to support breast feeding mothers in community are in place, more support needs to be established including regular assessment of weight to avoid hospital re-admissions.

\section{PITFALLS OF THE NEONATAL SCREENING PROGRAM FOR CONGENITAL ADRENAL HYPERPLASIA (CAH)}

doi:10.1136/archdischild-2012-302724.1761

HA Abu Hamdan, F Sabbagh, MM Abou Al-Seoud. Neonatal Division - Paediatric Department, King Fahad Armed Forces Hospital, Jeddah, Saudi Arabia

Background Prompt diagnosis and treatment of CAH is essential to prevent mortality and morbidity. The incidence of $\mathrm{CAH}$ ranges from 1 in 21270 (New Zealand) to 1 in 5000 (Saudi Arabia).

Our Aim:

Identify the incidence of $\mathrm{CAH}$ in our patient population.

Appraise the $\mathrm{CAH}$ screening program.

Method Retrospective analysis of filter paper blood samples from infants aged 36 hours or more collected from November 2007 - Sept 2011. The 17-hydroxyprogesterone (17-OHP) tests were part of metabolic screening program at KFAFH. Abnormal values were re-called.

Results 22381 of 22428 (99.7\%) births were screened for CAH. The turnaround time was 10 days. 124 infants [males 89 (72\%) females $31(28 \%)$ ] had abnormal levels and were re-called (recall rate $0.55 \%$ ). 76 infants had repeated serum 17-OHP concentration (response rate 61\%). 7 infants had abnormal elevated levels, 4 had ambiguouse genitalia(karyotype female), 3 male infants had symptoms of salt wasting at age of 9.10 and 37 days, turnaround time for the screening results was 11.9 and 16 days respectively. Delayed identification was due to failed contact and response in case 2 and 3 respectively. Recall failure was in 48/124 (39\%) reasons were wrong contact numbers in 36 cases $(75 \%)$ and no show in 10 cases $(21 \%)$. Failure to repeat in 2 preterm infants died secondary to prematurity.

Conclusion The incidence of CAH is 1 in 3333 in our patient population. Barriers for timely intervention were due to prolonged turnaround time, lack of family education. Strategies to improve the process should be implemented.

\section{CURRENT RESUSCITATION PRACTICES AMONG PEDIATRICIANS IN GUJARAT, INDIA - EFFECT OF 2010 RESUSCITATION GUIDELINES}

doi:10.1136/archdischild-2012-302724.1762

'AS Nimbalkar, 'SC Bansal, 'DV Patel, 'AR Sethi, 1,2SM Nimbalkar. 'Department of Pediatrics, Pramukhswami Medical College; 'Central Research Services, Charutar Arogya Mandal, Anand, India

Background and Aims Neonatal Resuscitation Guidelines are updated by international societies every five years. India does not have mandatory certification and hence many pediatricians may not change their practices which may be in variance with current guidelines. We aimed to see the acceptance and awareness of several recently incorporated practices in resuscitation.

Methods Questionnaire based cross-sectional study administered by a web based form the link of which was sent by email and/or social networking sites to 600 pediatricians in state of Gujarat. A total of 25 questions were asked requiring about 10 minutes of the respondents time.
Results 75 pediatricians responded with 62 usable questionnaires. About $83 \%$ participants worked in a Level $2 / 3$ NICU. $64 \%$ had resuscitated more than 20 neonates and $78 \%$ had attended more than 100 deliveries. Hence respondents were attending deliveries and required skills in resuscitation. $81.3 \%$ used bag and mask with only $33.3 \%$ using room air for resuscitation. $58.5 \%$ were using correct rate of ventilation. $76 \%$ had saturation monitors but only $17.2 \%$ had oxygen blenders. $31.7 \%$ approved of self inflating bag for free flow oxygen. $50 \%$ judged adequacy of ventilation by chest rise against $41.9 \%$ who used rising heart rate. Only about $50 \%$ pediatricians had undergone a training course in past 3 years. $41.7 \%$ believed in immediate cord cutting for normal deliveries. Only $32.3 \%$ responded correctly on the duration of resuscitation for asystole.

Conclusions The knowledge of pediatricians regarding resuscitation of neonates is poor. Only few pediatricians update themselves by attending courses.

\section{NEWBORN HEARING SCREENING: THE EXPERIENCE OF THE UNIVERSITY HOSPITAL OF MODENA}

doi:10.1136/archdischild-2012-302724.1763

${ }^{1} \mathrm{~S}$ Palma, ${ }^{2} \mathrm{MF}$ Roversi, ${ }^{3} \mathrm{FM}$ Artioli, ${ }^{3} \mathrm{G}$ Denti, ${ }^{2} \mathrm{~F}$ Ferrari, ${ }^{3} \mathrm{E}$ Genovese. ${ }^{1}$ Primary Care Department, Azienda ASL; ${ }^{2}$ Department of Neonatology, Neonatal Intensive Care Unit, Modena University; ${ }^{3}$ Ent Department, Audiology, Modena University, Modena, Italy

Background and Aims Newborn hearing screening programs allow to identify infants with bilateral permanent congenital hearing impairment and to facilitate early intervention to minimize the consequences on language development. In our country the concept of implementing universal newborn hearing screening protocols is still a topic of debate. Our aim was to analyze the first data collected in one year of experience since the adoption of the universal newborn hearing screening in Modena University Hospital.

Methods Data were collected during the period from $8^{\text {th }}$ April 2011 to $31^{\text {st }}$ March 2012. The screening was carried out by means of Transient Stimulus Evoked Otoacoustic Emissions, using, for well babies, a two-stage protocol: first screening stage on the second day after birth, followed by re-screening before discharge if a pass response was not obtained from both ears and a second stage follow up screening within 3 weeks later in case of failure. In case of a persistent failure response an audiologic evaluation was performed.

Results During the period of the study 3512 babies underwent newborn hearing screening, of these 203 presented increased risk factors. In well babies group, one child will undergo surgery for a cochlear implant, 3 children are receiving a prosthetic-rehabilitative treatment. The prevalence of permanent bilateral hearing loss resulted 1.2:1000.

Conclusion It is important to create an active collaboration between audiologists/ENT specialists and paediatricians to reach the objective of identifying infants with hearing loss as early as possible in order to implement early interventions.

\section{INVESTIGATION OF SOME ASPECTS OF PERCEIVED SOCIAL SUPPORT IN MOTHERS OF HOSPITALIZED CHILDREN IN NEONATAL INTENSIVE CARE UNIT}

doi:10.1136/archdischild-2012-302724.1764

'S Kara, ${ }^{2} \mathrm{~S}$ Tan, ${ }^{2} \mathrm{~S}$ Aldemir, ${ }^{3} \mathrm{AE}$ Y Ylmaz, ${ }^{3} \mathrm{MM}$ Tatll, ${ }^{4} \mathrm{U}$ Dilmen. 'Department of Neonatology, Ankara Training and Research Hospital; '2Department of Psychiatry; ${ }^{3}$ Department of Neonatology, Fatih University Medical Faculty; ${ }^{4}$ Department of Neonatology, Zekai Tahir Burak Maternity and Teaching Hospital, Ankara, Turkey

Purpose To identify the degree of perceived social support in mothers of hospitalized children in neonatal intensive care unit, and to assess in psychosocial aspects the relationship of the perceived social support with such variables as depression and anxiety level. 
Method A total of 50 mothers who have hospitalized infants in NICU, included in this study and the social support they perceived, and the their anxiety and depression levels were analyzed using "Multidimensional Scale of Perceived Social Support Scale" and Hospital Anxiety and Depression Scale (HAD scale), respectively.

Results Perceptions of total social support and the social support provided by friends in the mothers increased as the infants' birth weight decreased; the levels of total perceived social support and the perceived social support from families and spouses in the mothers getting pregnant with assisted conception techniques (ACT) were greater, compared with the mothers of spontaneous conception. The scores of total perceived social support and the perceived social support from families and spouses in depressed mothers were found to be lower than those in otherwise healthy mothers.

Conclusion It was concluded in our study that the mothers of the hospitalized infants in the neonatal intensive care unit required social support provided especially by the spouses and the families, disclosing the relationship of inadequate perception of such supports with development of depression.

\section{DO WE REALLY NEED TO REQUEST THAT BLOOD INVESTIGATION?}

doi:10.1136/archdischild-2012-302724.1765

AM Kulkarni, R Kinnapiran, N Kennea. Neonatology, St George's Hospital NHS Trust, London, UK

Blood investigations form an important aspect of patient management. Rationalisation of every test requested is needed to ensure optimum use of available resources. Wide variation in estimates of inappropriate laboratory use (4.5-95\%) has been reported in literature. Hospitalisation beyond 7 days, complex cases, level of staff training, lack of awareness of costs are factors contributing to laboratory over utilisation.

We assessed our blood investigation ordering practices in order to evaluate unnecessary investigations and clinical variance. We aimed to develop a standard framework for ordering investigations in preterm babies.

This retrospective study (Jan-June 2010) evaluated blood investigations requested in first 14 days of life on the all the babies born $<30$ weeks gestation and admitted to our neonatal unit. These data amounted to $\sim 5 \%$ of bed days on the unit over the study period. We created a standard investigation model for preterm babies and compared the findings with the standard.

37 babies were included in the study with total 479 cot days which represent $4.3 \%$ of unit cot days per year. We observed that we were requesting nearly $40 \%$ more tests as compared to standard. We estimated potential savings of $£ 1000 \mathrm{GBP}$ for only $4.3 \%$ of total cot days every year in our unit.

Following audit was presented in our departmental meeting for the education of junior members of the staff and to raise the awareness of the costs of investigations and the need to reduce unnecessary testing. We recommended colour coding of pathology forms according to cost.

\section{RANDOMISED CONTROLLED TRIAL OF POLYETHYLENE BAG AND EXOTHERMIC MATTRESS VERSUS POLYETHYLENE BAG ALONE FOR THERMOREGULATION IN PRETERM INFANTS AT BIRTH}

doi:10.1136/archdischild-2012-302724.1766

1,2,3LK McCarthy, ${ }^{1,2}$ EJ Molloy, ${ }^{1}$ AR Twomey, ${ }^{1} \mathrm{JF}$ Murphy, ${ }^{1,2,3} \mathrm{CPF}$ 0'Donnell. 'Department of Neonatology, The National Maternity Hospital; ${ }^{2}$ The National Children's Research Centre, Crumlin; ${ }^{3}$ University College Dublin, Dublin, Ireland

Aims To determine whether placing very preterm infants in polyethylene bags( $\mathrm{PB})$ and on exothermic mattresses(EM) in the delivery room(DR) results in more infants with temperatures of $36.5-37.5^{\circ} \mathrm{C}$ on NICU admission.

Methods Infants $<31$ weeks' were randomised to treatment with or without EM in DR. All infants were placed in $\mathrm{PB}$ under radiant heaters and were transferred to the NICU in transport incubators. Infants' rectal temperature was measured on admission. We estimated we would need to recruit 118 infants.

Results The external data safety monitoring committee recommended stopping recruitment after analyzing data from 59 infants due to a significant difference in primary outcome between the groups. We present data for 72 infants enrolled when this recommendation was made. Fewer infants randomised to EM had temperatures within the target range $[41 \% \vee 77 \%, p=0.002]$ and more had admission temperature $>37.5 \mathrm{C}$ [ $46 \%$ v $17 \%, \mathrm{p}=0.009]$.

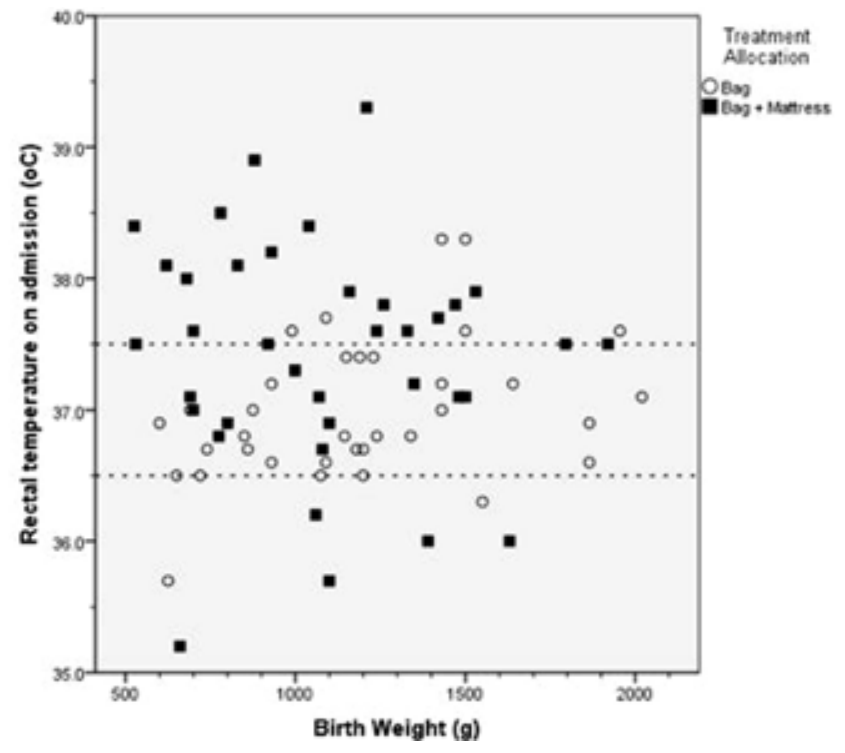

Abstract 1766 Figure 1

Abstract 1766 Table 1 Results

\begin{tabular}{lccc}
\hline [Data are * mean $(\mathbf{S D})$ or \#n (\%)] & BAG \& MATTRESS (N=37) & BAG (N=35) & P value \\
\hline Gestational age $(w k s)^{*}$ & $28(2)$ & $28(2)$ & 0.584 \\
Birth weight $(\mathrm{g})^{*}$ & $1085(360)$ & $1194(386)$ & 0.222 \\
Adm rectal temp 36.5-37.5(C)\# & $15(41)$ & $27(77)$ & 0.002 \\
Adm rectal temp (C) & $37.4(0.9)$ & $37.0(0.5)$ & 0.017 \\
Adm rectal temp>37.5C\# & $17(46)$ & $6(17)$ & 0.009 \\
Adm rectal temp<36.5C\# & $5(14)$ & $2(6)$ & 0.264 \\
\hline
\end{tabular}

Conclusions In newborn very preterm infants, using EM in addition to $\mathrm{PB}$ in the $\mathrm{DR}$ resulted in more infants with temperatures outside normal range and more hyperthermia on admission to NICU.

\section{VALIDATION OF THE DUTCH 60 MONTHS AGES AND STAGES OUESTIONNAIRE (ASO)}

doi:10.1136/archdischild-2012-302724.1767

1J Hornman, ${ }^{1} \mathrm{JM}$ Kerstjens, ${ }^{2} \mathrm{AF}$ de Winter, ${ }^{1} \mathrm{AF}$ Bos, ${ }^{2} \mathrm{SA}$ Reijneveld, Lollipop Study. ${ }^{1}$ Department of Neonatology, Beatrix's Children Hospital, University Medical Centre Groningen; ${ }^{2}$ Department of Health Sciences, University Medical Centre Groningen, University of Groningen, Groningen, The Netherlands

Introduction The Ages and Stages Questionnaire (ASQ) is currently the most widely used parent-completed developmental screener. Psychometric properties of the Dutch ASQ 60-months version (Dutch_ASQ-60) have not yet been assessed. 